\title{
Psychometric Data for the Reliability and Validity of the Mini Tinnitus Questionnaire Turkish Version (TQ 12-T)
}

\author{
Emre Gürses ${ }^{1}$, Banu Müjdeci², Songül Aksoy ${ }^{1}$ \\ 1 Department of Audiology, Hacettepe University, Turkey; \\ 2 Department of Audiology, Ankara Ylldırım Beyazıt University, Turkey; \\ Emre Gürses, ORCID: 0000-0002-5157-7227 \\ Banu Müjdeci, ORCID: 0000-0002-3660-3650 \\ Songül Aksoy, ORCID: 0000-0003-4584-5528
}

\begin{abstract}
Objective: To investigate the reliability and validity of the Turkish version of the Mini-Tinnitus Questionnaire (TQ 12-T).

Methods: The study consisted of 120 patients with tinnitus $(60 \mathrm{~F}+60 \mathrm{M}$; Mean Age: $46.8 \pm 15.1$ years). Audiological evaluation, tinnitus mapping, tinnitus handicap inventory (THI) and TQ 12-T were applied to the participants. Internal consistency and reliability were assessed by Cronbach's alpha. To evaluate the accuracy of the original findings, a retest was performed with $20 \%$ of the participants. The validity of the TQ 12-T was assessed, analyzing the correlation with THI. We assessed construct validity by analyzing the patients according to their age and tinnitus duration.
\end{abstract}

Results: High internal consistency, reliability $(\alpha=0.87)$ and a high intraclass correlation coefficient (ICC 0.96, p < 0.001 ) were found. The internal consistency reliabilities of the two subscales were $\alpha=0.74$ and $\alpha=0.84$ for "health anxiety" and "cognitive distress," respectively. There was a strong positive correlation between THI and TQ 12-T total scores $(r=0.985, p<0.01)$.

Conclusion: TQ 12-T is a valid, reliable and brief questionnaire. TQ 12-T could easily be used alongside other tinnitus or health-related questionnaires since it takes only a few minutes to complete.

Keywords: Tinnitus, Mini-Tinnitus Questionnaire, reliability and validity

\section{Correspondence: Emre Gürses}

Department of Audiology, Faculty of Health Science, Hacettepe University, 06100 Ankara, Turkey.

E-mail: gursess.emre@gmail.com

Received: 28.5.2020; Accepted: 13.7.2020
Online available at: www.entupdatesjournal.org 


\section{Introduction}

Tinnitus is a subjective perception in the absence of any external stimulus. ${ }^{[1]}$ The prevalence of tinnitus is approximately $4.6 \%$ to $30 \%$ of the general adult population across various nationalities and it increases with age..$^{[2-5]}$ Approximately $20 \%$ of people with tinnitus suggest that this symptom affects their quality of life. ${ }^{[6,7]}$ Tinnitus causes deterioration in daily life activities, sleep, mood and concentration in about $0.5-3 \%$ of the general population. ${ }^{[6,8]}$ Because there is no objective and definitive test to ascertain its effect on a patient, determining the multidimensional impact of subjective tinnitus has crucial importance.

Two primary goals should be taken into consideration regarding the approach towards tinnitus treatment: habituation of the reaction to tinnitus while aiming to reduce the physiological effects caused by tinnitus (such as depression, anxiety, insomnia, etc.) and habituation of the perception of tinnitus while reducing or eliminating the sounds sensed by the individuals. ${ }^{[9]}$ Recently, although there are practical and successful treatment methods for the former, psychometric instruments are needed to understand the causes of distress. Moreover, to determine and follow the effectiveness of tinnitus treatment and therapy, psychometric instruments are indispensable.

The Tinnitus Research Initiative has created a consensus with leading researchers from 11 countries to determine the minimum requirements to be considered in evaluations and measurements. ${ }^{[10]}$ The importance of requirements were marked as level A "essential”, level B "highly recommended" and level C "might be of interest". Validated questionnaires for the assessment of tinnitus severity were determined as essential. The committee has suggested the use of some of the worldwide questionnaires, such as the Tinnitus Handicap Inventory (THI) and Tinnitus Questionnaire (TQ).

The TQ and THI are frequently used as reliable questionnaires to assess the psychological effects of tinnitus-related disturbances. The TQ has 52 items and five subscales, whereas the THI has 25 items and three subscales to assess tinnitus distress. Although performing both of these questionnaires provides versatile information to understand the nature of tinnitus, they are time-consuming in clinical practice. Therefore, a shortened version of the TQ (i.e., TQ 12) was developed by Hiller and Goebel. ${ }^{[1]}$ TQ 12, called Mini TQ, is easy and faster to administer in a clinical setting. The questionnaire, which takes about 2-3 minutes to complete, has similar psychometric features compared to more complex questionnaires. ${ }^{[12]}$

The Mini TQ may help to quantify tinnitus distress by defining grades of tinnitus symptoms and following the improvement in tinnitus distress after treatment or therapy as part of the overall tinnitus rehabilitation. So far, the Mini TQ has been translated into several languages, including Portuguese ${ }^{[12]}$, Greek ${ }^{[13]}$, Arabic ${ }^{[14]}$, Dutch ${ }^{[15]}$, Chinese ${ }^{[16]}$, Malay ${ }^{[17]}$ and Italian. ${ }^{[18]}$ However, there is still a shortage of translated versions of the TQ 12 to be accepted as a worldwide questionnaire. The purpose of this study was to investigate the reliability and validity of the Turkish version of the Mini-Tinnitus Questionnaire (TQ 12-T).

\section{Materials and Methods}

\section{Participants}

The current study included 120 (Mean: $46.8 \pm 15.1$ years) individuals with tinnitus. Following approval of the ethical committee of Hacettepe University (Project number: GO 19/486), all individuals completed the evaluation forms. The data obtained from the evaluation forms were age, sex, location and duration and features of tinnitus (pulsatile or non-pulsatile). Inclusion criteria were determined as: age between 18 and 65 years, tinnitus experienced for at least for three months and no apparent communication impairment. A short interview was also performed to determine the previous history of tinnitus-related disease, previous hearing tests and treatments, and physical examination of bruxism and neck movements. Almost all of the study's data collection methodology was also part of a clinical routine including pure tone and speech audiometry, immittance measurement, tone decay, reflex decay testing and tinnitus mapping. In addition, a eustachian function test was applied if needed. More than $10 \mathrm{~dB}$ differences for at least three contiguous frequencies were accepted as an asymmetric hearing loss in the pure-tone evaluation. For determining the tinnitus location, participants were asked where they heard their tinnitus. Tinnitus pitch was marked as low, middle, high and very high frequency since the tinnitus pitch was found below $500 \mathrm{~Hz}$, between $500 \mathrm{~Hz}$ to $2 \mathrm{kHz}$, higher than $2 \mathrm{kHz}-8 \mathrm{kHz}$ and higher than $8 \mathrm{kHz}$, respectively. 


\section{Audiological Evaluation and Tinnitus Mapping}

Following otolaryngologic examination, air (0.25 to 14 $\mathrm{kHz})$ and bone $(0.5$ to $4 \mathrm{kHz})$ thresholds were measured in both ears in a double-walled sound-isolated booth. The tests were carried out according to a modified Hughson-Westlake procedure using a GSI 61 clinical audiometer (Grason-Stadler, Eden Prairie, MN) and TDH-39 supra-aural earphones (Telephonics, Farmingdale, NY). HDA 200 circum-aural earphones (Sennheiser, Old Lyme, CT) and a bone vibrator (Radioear Corp, New Eagle, PA) were used for high frequencies over $8 \mathrm{kHz}$ and bone conduction threshold, respectively. Hearing loss degree was determined according to the classification of Clark JG ${ }^{[19]}$ with mean frequencies of $0.5,1,2$ and $4 \mathrm{kHz}$. Additional information such as hearing asymmetry and high-frequency hearing loss were noted. Immitancemetry was done only using a 226-Hz probe tone emitted by a GSI Tympstar tympanometer (Grason-Stadler, Eden Prairie, MN).

Tinnitus-matching tones were delivered to the contralateral and ipsilateral ear for unilateral and bilateral tinnitus, respectively. The clinician provided instructions verbally and presented two stimuli to the subject using manual control. Each participant was asked to identify a similar frequency with their tinnitus. The identical frequency was determined gradually through two different stimuli selections.

\section{Tinnitus Questionnaire Short Form (TQ 12)}

Unlike the TQ, the Mini TQ is brief and short with 12 items selected from TQ subscales (emotional distress; 1, 2, 5, 6, 7, 9, 12; cognitive distress: 3, 10, 11; sleep disturbance: 8). Individuals mark their distress level as "True", "Part- ly True" or "Not True". Scores of the three-point Likert Scale are calculated as 0 points (Not True), 1 point (Partly True) and 2 points (True). The total score is from 0 to 24 points. The grading system, according to an overall score for the TQ 12, is shown in Table 1.

\section{Adapting the Turkish Tinnitus Questionnaire Short Form (TQ12-T)}

The Turkish Tinnitus Questionnaire Short Form (TQ12-T) was developed with a "back translation" method. Initially, the TQ12 was translated into Turkish independently by a bilingual physician from the original language after obtaining written consent from the developer (i.e., Hiller and Goebel). Then an English lecturer re-translated this Turkish version back into the original language. After that, a bilingual lecturer different from the others compared the translations to the original TQ12. After completing the translation procedure, ten individuals without any academic title and two clinicians were convened to ensure the questionnaire reflected common and daily language. The Turkish version of the TQ 12 was finalized with minor revisions.

\section{Tinnitus Handicap Inventory (THI)}

The THI is a well-known self-assessment tool to assess tinnitus distress in clinical practice. The THI has 25 items and three subscales, which are functional, emotional and catastrophic. Individuals mark their distress level as "Yes", "No", "Sometimes" and respective scores such as 4, 0 and 2 are given. Distress grading is shown in Table 2. Good reliability and validity were reported for the total score of the original and all different language adapted versions. ${ }^{[20-22]}$

Table 1. Grading System according to total score for the TQ 12.

\begin{tabular}{ccc} 
Total Score & \multicolumn{1}{c}{ Classification } & Grade \\
$0-7$ & No clinically relevant distress due to tinnitus & Distress Level I \\
$8-12$ & Moderately distressed & Distress Level II \\
$13-18$ & Severely distressed & Distress Level III \\
$19-24$ & Most severely distressed & Distress Level IV
\end{tabular}

TQ 12=Tinnitus Questionnaire Short Form 
Table 2. Grading System according to the total score for the THI.

\begin{tabular}{ccc}
\hline Total Score & Classification & Grade \\
\hline $0-16$ & Slight & 1 \\
$18-36$ & Mild & 2 \\
$38-56$ & Moderate & 3 \\
$58-76$ & Severe & 4 \\
$78-100$ & Catastrophic & 5
\end{tabular}

THI: Tinnitus Handicap Inventory

\section{Statistical Analysis}

IBM SPSS Statistics software version 23 (IBMC) Corp., Armonk, NY) was used for the statistical analyses. Psycho-metric values are presented using mean, standard deviation and item-total correlations. The internal consistency of the TQ 12-T was assessed using Cronbach's alpha. Sampling adequacy was measured by Kaiser-Meyer-Olkin (KMO). To determine the ideal number of factors, Scree plots and Eigenvalues were performed. The test-retest reliability was examined using the intraclass correlation coefficient (ICC). Correlations between TQ 12-T and THI were analyzed for convergent validity using a two-sided Pearson correlation test. An overall 5\% Type-I error level was used to infer statistical significance. A p value less than 0.05 was considered statistically significant.

\section{Results}

Descriptive results of the 120 individuals $(60 \mathrm{~F}+60 \mathrm{M}$; Mean Age: $46.8 \pm 15.1$ years), such as tinnitus duration, location, pitch, hearing loss degree and asymmetry are shown in Table 3. The mean tinnitus duration of the participants was $5.10 \pm 6.14$ years. The mean THI was $54.40 \pm 24.34$. Although $57(47.5 \%)$ of the participants had normal hearing according to pure-tone average, 17 participants had high-frequency hearing loss, 4 participants had an acoustic notch and 1 participant had low-frequency hearing loss. Six of the total participants had bruxism history. One participant was a cochlear implant user. More details about the participants are shown in table 3. No significant differences were found in TQ 12-T scores in terms of gender $(\mathrm{p}=0.16)$ and tinnitus location $(\mathrm{p}=0.85)$.

\section{Psychometric Values}

The mean TQ 12-T total score was $12.98 \pm 5.95$. The item-total correlation of each item is shown in Table 4. No floor or ceiling effects were found. Bartlett's test of sphericity was found to be statistically significant $\left(\mathrm{X}^{2}=525.65\right.$, $\mathrm{p}<0.001)$. To verify sampling rate adequacy, the KaiserMeyer-Olkin measure was performed. The results of KMO (0.876) confirmed the sampling adequacy. Eigenvalue and scree plot curves were used to determine factors with a high correlation of TQ 12-T test items. Eigenvalue values of factors greater than one were evaluated (Figure 1). The eigenvalues showed that the first (42.18\%) and the second $(11.13 \%)$ factors explained the variability. The two-factor solution explained $53.12 \%$ of the cumulative variance. Therefore, remaining analyses were performed

Table 3. Descriptive properties of the participants.

\begin{tabular}{|c|c|c|c|c|}
\hline Hearing Loss Degree & Hearing Asymmetry & Tinnitus Location & Tinnitus Pitch & Pulsatil Tinnitus \\
\hline N (\%) & N (\%) & N (\%) & $N(\%)$ & N (\%) \\
\hline \multicolumn{5}{|l|}{ Normal: 57 (47.5\%) } \\
\hline Slight: 26 (21.6\%) & & Right: 21 (17.5\%) & Low: $16(13.3 \%)$ & \\
\hline Mild: 19 (15.8\%) & Yes: 39 (32.5\%) & Left: $44(36.6 \%)$ & Medium: $17(14.12 \%)$ & Yes: $9(7.5 \%)$ \\
\hline \multicolumn{5}{|l|}{ Moderate: $10(8.3 \%)$} \\
\hline Mod-Severe: 2 (1.6\%) & No: $81(67.5 \%)$ & Both Ears: 43 (35.8\%) & High: 65 (54.1\%) Very & No: $111(92.5 \%)$ \\
\hline Severe: 2 (1.6\%) & & Head:10 (8.3\%) & High: $7(5.83 \%)$ & \\
\hline \multicolumn{5}{|l|}{ Profound: $1(0.8 \%)$} \\
\hline SSD: $3(2.5 \%)$ & & Missing: $2(1.6 \%)$ & Missing: $15(12.5 \%)$ & \\
\hline
\end{tabular}

SSD: Single side deafness, Mod-severe: Moderate-severe, N: Number of patients 
Table 4. Mean, SD and variance of the TQ 12-T.

\begin{tabular}{|c|c|c|c|}
\hline & Mean & SD & Item-Total Correlation \\
\hline 1. I am aware of the noises from the moment I get up to the moment I sleep & 1.54 & 0.64 & 0.59 \\
\hline $\begin{array}{l}\text { 2. Because of the noises I worry that there is something seriously wrong with } \\
\text { my body }\end{array}$ & 0.70 & 0.78 & 0.46 \\
\hline 3. If the noises continue my life will not be worth living & 0.45 & 0.69 & 0.46 \\
\hline 4. I am more irritable with my family and friends because of the noises & 0.60 & 0.81 & 0.54 \\
\hline 5. I worry that the noises might damage my physical health & 1.05 & 0.79 & 0.48 \\
\hline 6. I find it harder to relax because of the noises & 1.39 & 0.70 & 0.53 \\
\hline 7. My noises are often so bad that I cannot ignore them & 1.26 & 0.77 & 0.70 \\
\hline 8. It takes me longer to get to sleep because of the noises & 1.25 & 0.82 & 0.42 \\
\hline 9. I am more liable to feel low because of the noises & 1.15 & 0.84 & 0.69 \\
\hline 10. I often think about whether the noises will ever go away & 1.49 & 0.73 & 0.59 \\
\hline 11. I am a victim of my noises & 0.83 & 0.86 & 0.60 \\
\hline 12. The noises have affected my concentration & 1.33 & 0.71 & 0.60 \\
\hline
\end{tabular}

TQ 12-T: Turkish Tinnitus Questionnaire Short Form, SD: Standart Deviation

Table 5. Results of a rotated component matrix.

\begin{tabular}{|c|c|c|}
\hline \multirow{2}{*}{ Items } & \multicolumn{2}{|c|}{ Subscales } \\
\hline & Factor I & Factor II \\
\hline $\begin{array}{l}\text { 1. I am aware of the noises from the moment I get up to the moment I } \\
\text { sleep }\end{array}$ & 0.16 & 0.74 \\
\hline $\begin{array}{l}\text { 2. Because of the noises I worry that there is something seriously wrong } \\
\text { with my body }\end{array}$ & 0.54 & 0.24 \\
\hline 3. If the noises continue my life will not be worth living & 0.77 & 0.09 \\
\hline 4. I am more irritable with my family and friends because of the noises & 0.70 & 0.24 \\
\hline 5. I worry that the noises might damage my physical health & 0.76 & 0.11 \\
\hline 6. I find it harder to relax because of the noises & 0.26 & 0.60 \\
\hline 7. My noises are often so bad that I cannot ignore them & 0.21 & 0.83 \\
\hline 8. It takes me longer to get to sleep because of the noises & -0.02 & 0.66 \\
\hline 9. I am more liable to feel low because of the noises & 0.65 & 0.41 \\
\hline 10. I often think about whether the noises will ever go away & 0.34 & 0.59 \\
\hline 11. I am a victim of my noises & 0.49 & 0.47 \\
\hline 12. The noises have affected my concentration & 0.25 & 0.68 \\
\hline
\end{tabular}




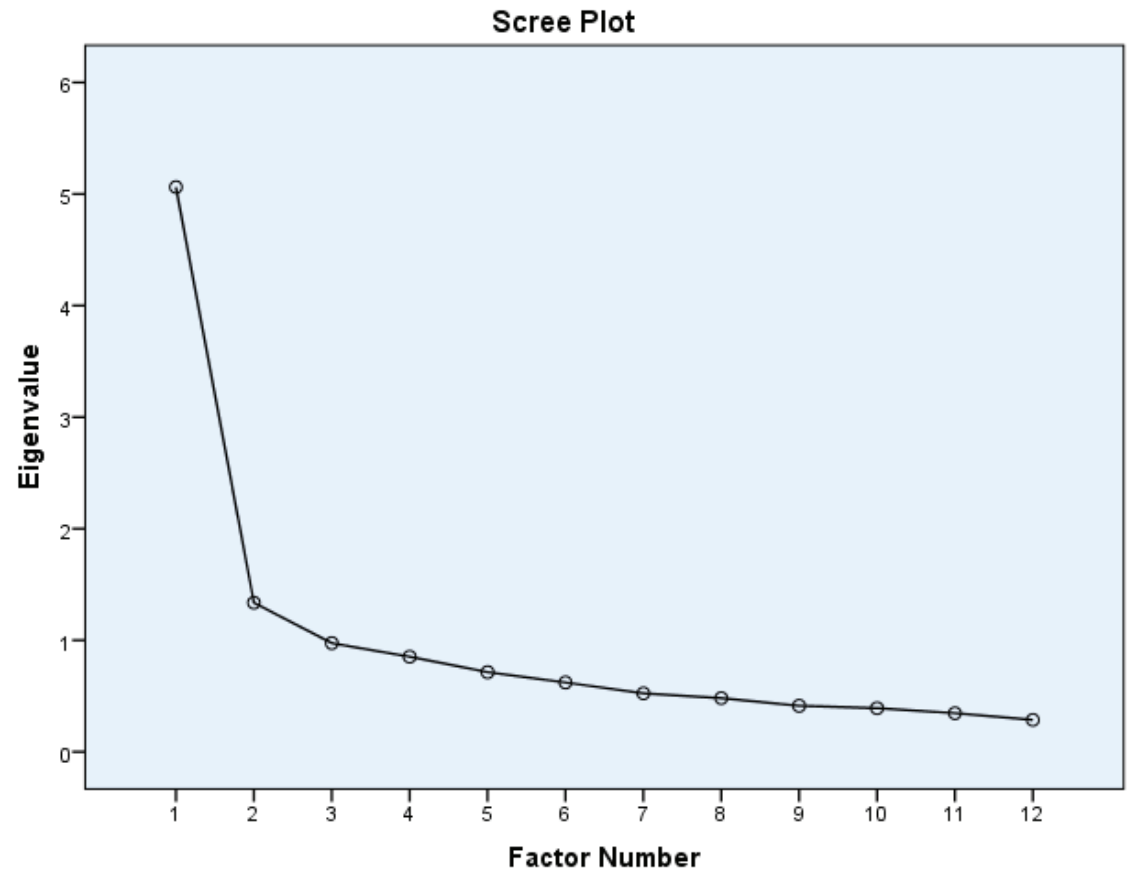

Figure 1. Scree Plot for the TQ 12-T.

for a two-factor solution. Table 5 shows rotated component matrix results to determine items and factor relations (factor 1: health anxiety, factor 2: cognitive distress).

\section{Reliability and Validity of the TQ 12-T}

The reliability of the TQ 12-T was evaluated according to its internal consistency and its test-retest reliability. The internal consistency reliability for the total scale, as assessed by Cronbach's $\alpha$, was 0.87 . The internal consistency reliabilities of the two subscales were 0.74 and 0.84 for health anxiety and cognitive distress, respectively.

Twenty percent of the participants were asked to refill the questionnaire after 2-3 days for the test-retest reliability. Results showed that the intra-class correlation coefficiency was statistically significant $(0.96, \mathrm{p}<0.001)$.

The correlation between THI and TQ12-T was evaluated both for total and subscale scores using Pearson correlation analyses. There was a strong positive correlation between THI and TQ 12-T total scores $(r=0.985, \mathrm{p}<0.01)$. A scatterplot is summarized in Figure 2. It was found that all subscales were significantly well correlated. The strongest correlation was between the health anxiety subscale of TQ
$12-\mathrm{T}$ and the emotional subscale of THI $(\mathrm{r}=0.789, \mathrm{p}<0.01)$. In addition, a positive moderate correlation was found between the cognitive distress subscale of TQ 12-T and both catastrophic $(\mathrm{r}=0.638, \mathrm{p}<0.01)$ and functional $(\mathrm{r}=0.558$, $\mathrm{p}<0.01)$ subscales of the THI. No statistically significant correlation was found $(\mathrm{p}=0.63)$ between tinnitus duration and TQ 12-T total scores.

\section{Discussion}

Perception of tinnitus severity is associated with psychological factors and general health factors. ${ }^{[23]}$ The main purpose of tinnitus treatment is to reduce the effect of tinnitus and increase the individual's quality of life. Therefore, valid and reliable methods are needed to evaluate the effects of tinnitus. ${ }^{[1]}$ In the present study, we aimed to translate Mini TQ into Turkish and investigate the validity and reliability of the TQ 12-T.

There are many questionnaires evaluating tinnitus-induced distress. ${ }^{[24]}$ The Mini-TQ is easy and quick to administer and also has good psychometric properties. This questionnaire has not been adapted to Turkish to date. It took about three minutes to complete the TQ 12-T for 


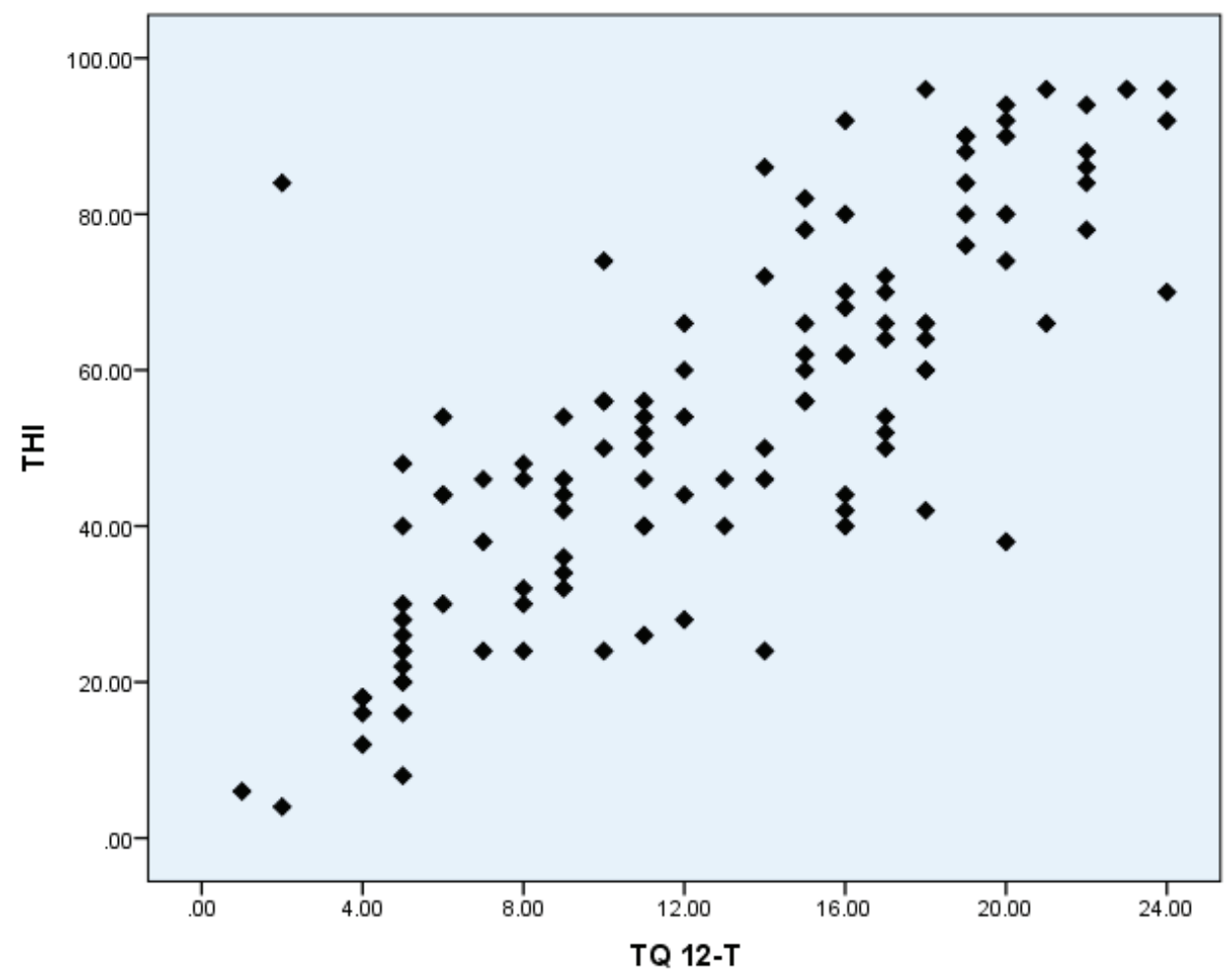

Figure 2. Scatterplot between THI and TQ 12-T.

the individuals who participated in the present study. Additionally, it is stated that the Mini TQ does not have psychometric disadvantages compared to the full TQ. ${ }^{25]}$

The mean TQ 12-T total score was $12.98 \pm 5.95$. So far, mean scores have been found from 7.95 \pm 5.57 (Greek versions) to $15.4 \pm 5.7$ (German versions). Our results can be accepted as compatible with the literature. According to the grading system, our data were equal to distress level III and grade III (Moderate) in TQ 12-T and THI, respectively. Similar distress levels could be accepted as proof of the validity of TQ 12-T.

The original Mini TQ items were adapted from 3 factors of the TQ, "emotional distress", "intrusiveness" and "cognitive distress". In Malay and Arabic versions, no factor solution was suggested. According to the validation of the Greek population, three factors were proposed, namely "indicative of distress", "indicative of health pre-occupation" and "indicative of depression". In our study, a statistically two-factor solution was determined, which is corroborated by the Italian version. Factor naming is the main issue for TQ 12 adaptation studies since a different number of subtests were defined in previous studies. After thorough content analysis of the items by three researchers and the similarity of factor loadings with the Italian version, two factors were named "Factor I=health anxiety" and "Factor II=cognitive distress" in the TQ 12-T.

The results of our study showed good internal consistency, test-retest reliability and clinical validity for the TQ 12-T. Internal consistency was excellent $(>0.70)$ for TQ 12 T. For validation purposes, the Mini TQ was performed to correlate with other questionnaires such as the Perceived Stress Scale (PSS 14) ${ }^{[13]}$, Multidimensional Health Locus of Control Scale (MHLoC) ${ }^{[13]}$, Brief Symptom Inventory (BSI) ${ }^{[18]}$, Short Form Health Survey (SF 36) ${ }^{[18]}$, TQ ${ }^{[15]}$ and THI ${ }^{[17,18]}$ in previous studies. All questionnaires showed good correlation with TQ 12. In our study, a strong correlation was found between the TQ 12-T and THI ( $\mathrm{r}=0.98)$, whereas the Italian version found a moderate correlation $(\mathrm{r}=0.65)$. Our correlation results corroborate the Malay version $(\mathrm{r}=0.83)$. So far, only the Italian version provided subtest correlations between THI and Mini TQ. We found that all subtest correlations were significant. The strongest correlation of all was found between the functional subscale of THI and Cognitive Distress of TQ 12. In our study, 
we also performed correlation analyses among subtests of THI and TQ 12. All subtests showed a statistically significant correlation. The strongest correlation was between the health anxiety subscale of TQ 12-T and the emotional subscale of THI $(r=0.789)$. Although subscale loadings were almost the same as the Italian version, the strongest correlation was different between THI and TQ 12. This can be explained by the mean age and duration of tinnitus differences in the studies. Overall, these results showed the validity of the Turkish version of the Mini TQ. In addition, the Mini TQ is suitable to be used alongside other tinnitus or health-related questionnaires since it takes only a few minutes to complete.

Our findings, as discussed above, confirmed previous study findings evaluating the Mini TQ. In these studies, similar to our findings, the Mini TQ was found to be a valid and reliable questionnaire with good internal consistency both for total score and subscales. Cerejeira et al. ${ }^{[12]}$ found that the Portuguese version of the Mini TQ is as suitable as the English version. They stated that the questionnaire could be used in routine practice, initial evaluations and follow-up in clinics. Hiller and Goebel ${ }^{[11]}$ stated that the Mini TQ might be more valuable for individuals with chronic tinnitus due to the temporary psychological problems in the acute period.

\section{Conclusion}

As far as we know, TQ 12-T is the second validated tinnitus-related questionnaire for a Turkish speaking population, after the well-known THI. In our study, we found the TQ 12-T to be a valid and reliable questionnaire for assessing tinnitus-related-stress. This questionnaire can be easily applied to tinnitus patients who speak Turkish in the clinical setting for routine assessment. The use of the questionnaire in initial evaluations and follow-up can provide information in evaluating the effectiveness of treatment. In addition, the TQ 12-T could easily be used alongside other tinnitus or health-related questionnaires since it takes only a few minutes to complete.

\section{Acknowledgements: None}

Ethics Committee Approval: The study protocol was approved by the Ethics Committee of Hacettepe University (Project no: GO 19/486).

Informed Consent: Informed consent was obtained from all individual participants included in the study.

Author Contributions: Designing the study - E.G.; Collecting the data -E.G., B.M., S.A.; Analysing the data -E.G., S.A.; Writing the manuscript - E.G., B.M..; Confirming the accuracy of the data and the analyses - B.M., S.A.

Conflict of Interest: The authors have no conflicts of interest to declare.

Financial Disclosure: The authors declare that the present study has received no financial support. 


\section{References}

1. Hallam R, Rachman S, Hinchcliffe R. Psychological aspects of tinnitus. Contributions to Medical Psychology 1984;3:31-53.

2. Bogo R, Farah A, Karlsson KK, Pedersen NL, Svartengren M, Skjönsberg Å. Prevalence, Incidence Proportion, and Heritability for Tinnitus: A Longitudinal Twin Study. Ear Hear 2017;38:292-300.

3. Kim HJ, Lee HJ, An SY, et al. Analysis of the prevalence and associated risk factors of tinnitus in adults. PLoS One 2015;10:e127578.

4. Jalessi M, Farhadi M, Asghari A, et al. Tinnitus: an epidemiologic study in Iranian population. Acta Med Iran 2013;51:886-91.

5. Park KH, Lee SH, Koo JW, et al. Prevalence and associated factors of tinnitus: data from the Korean National Health and Nutrition Examination Survey 2009-2011. J Epidemiol. 2014;24:417-26.

6. Adrian D, El Refaie A. The epidemiology of tinnitus. In Tyler R, editör. The Handbook of Tinnitus. San Diego, CA: Singular Publishing Group. 2000. p. 1-23.

7. Coles R. Epidemiology of tinnitus:(2) Demographic and clinical features. J Laryngol Otol 1984;98:195-202.

8. Axelsson A, Ringdahl A. Tinnitus--a study of its prevalence and characteristics. Br J Audiol 1989;23:53-62.

9. Jastreboff PJ, Jastreboff MM. Tinnitus Retraining Therapy (TRT) as a method for treatment of tinnitus and hyperacusis patients. J Am Acad Audiol 2000;11:162-77.

10. Langguth B, Goodey R, Azevedo A, et al. Consensus for tinnitus patient assessment and treatment outcome measurement: Tinnitus Research Initiative meeting, Regensburg, July 2006. Prog Brain Res 2007;166:52536.

11. Hiller W, Goebel G. Rapid assessment of tinnitus-related psychological distress using the Mini-TQ. Int J Audiol 2004;43:600-4.

12. Cerejeira R, Cerejeira J, Paiva S, et al. The Portuguese version of Mini-Tinnitus Questionnaire: brief screening test for assessment of tinnitus-induced stress. Otol Neurotol 2009;30:112-5.

13. Panagiotopoulos G, Galanakis M, Varvogli L, Chrousos G, Darviri C. Validation of the Greek version of Mini Tinnitus Questionnaire as a Brief Screening Test for Assessment of Tinnitus-related Distress: our experience in 301 adult patients. Clin Otolaryngol 2015;40:363-9.
14. El Beaino M, Eter E. Arabic validation of the tinnitus handicap inventory and the mini-tinnitus questionnaire on 100 adult patients. Clin Otolaryngol 2018;43:377-82.

15. Vanneste S, Plazier M, van der Loo E, et al. Validation of the Mini-TQ in a Dutch-speaking population: a rapid assessment for tinnitus-related distress. B-ENT 2011;7:31-6.

16. Kam AC. A screening tool for tinnitus-related distress--the Chinese version of Mini Tinnitus Questionnaire: our experience in one hundred and fourteen adult patients. Clin Otolaryngol 2012;37:234-7.

17. Ewe S, Ishak WS, Aniza I, Asma A. The Tinnitus Handicap Inventory \& Mini-Tinnitus Questionnaires: Reliability \& Validity of the Malay Version. Int Med J 2019;26:522-4.

18. Moschen R, Fioretti A, Eibenstein A, et al. Validation of the Italian Tinnitus Questionnaire Short Form (TQ 12-I) as a Brief Test for the Assessment of Tinnitus-Related Distress: Results of a Cross-Sectional Multicenter-Study. Front Psychol 2018;9:65.

19. Clark JG. Uses and abuses of hearing loss classification. ASHA 1981;23:493-500

20. Newman CW, Jacobson GP, Spitzer JB. Development of the Tinnitus Handicap Inventory. Arch Otolaryngol Head Neck Surg 1996;122:143-8.

21. Passi S, Ralli G, Capparelli E, Mammone A, Scacciatelli D, Cianfrone G. The THI questionnaire: psychometric data for reliability and validity of the Italian version. Int Tinnitus J 2008;14:26-33.

22. Aksoy S, Firat Y, Alpar R. The Tinnitus Handicap Inventory: a study of validity and reliability. Int Tinnitus J 2007;13:94-8.

23. Baskill J, Coles R, Lutman M, Axelsson A. Tinnitus severity grading: longitudinal studies. Proc IVth Int Tinnitus Semin Amsterdam/New York: Kugler Publ 1991. p. 457-60.

24. Zeman F, Koller M, Schecklmann M, Langguth B, Landgrebe M; TRI database study group. Tinnitus assessment by means of standardized self-report questionnaires: psychometric properties of the Tinnitus Questionnaire (TQ), the Tinnitus Handicap Inventory (THI), and their short versions in an international and multi-lingual sample. Health Qual Life Outcomes 2012;10:128.

25. Baguley DM, Humphriss RL, Hodgson CA. Convergent validity of the tinnitus handicap inventory and the tinnitus questionnaire. J Laryngol Otol 2000;114:840-3.

This is an open access article distributed under the terms of the Creative Commons Attribution-NonCommercial-NoDerivs 3.0 Unported (CC BY- NC-ND3.0) Licence (http://creativecommons.org/licenses/by-nc-nd/3.0/) which permits unrestricted noncommercial use, distribution, and reproduction in any medium, provided the original work is properly cited.

Please cite this article as: Gürses E, Müjdeci B, Aksoy S. Psychometric Data for the Reliability and Validity of the Mini Tinnitus Questionnaire Turkish Version (TQ 12-T). ENT Updates 2020;10(2): 340-348. 